\title{
Normal modes in orthorhombic media
}

\author{
Yuriy Ivanov ${ }^{\oplus},{ }^{1}$ Alexey Stovas ${ }^{1}$ and Vladimir Kazei ${ }^{\circledR 2}$ \\ ${ }^{1}$ Department of Geoscience and Petroleum, Norwegian University of Science and Technology, NO-7491 Trondheim, Norway. E-mail: yuriy.ivanov@ntnu.no \\ ${ }^{2}$ King Abdulah University of Science and Technology, Thuwal, Saudi-Arabia
}

Accepted 2018 December 20. Received 2018 December 15; in original form 2017 November 14

\begin{abstract}
SUMMAR Y
Guided waves in a water layer overlaying an elastic half-space are known as normal modes. They are often present in seismic recordings at long offsets in shallow-water environment and generally considered coherent noise. The normal modes, however, carry important information about the near-surface and, as demonstrated by a number of authors, can be used to obtain the shallow velocity model. There is a growing evidence that the latter needs not to be isotropic due to various geological reasons. Motivated by that, we consider the normal-mode propagation in case the elastic half-space exhibits orthorhombic anisotropy. We derive the period equation that describes the normal-mode phase velocity dispersion. To simplify the complicated expression, we present acoustic and ellipsoidal orthorhombic approximations. We also outline the approach towards the group velocity and group azimuth calculation and apply it to the ellipsoidal case to obtain concise and intuitive expressions. Using numerical test, we study the relation between phase and group domains in elastic orthorhombic case. The deviation between velocities and azimuths in these domains is the strongest for low frequencies and it rapidly decreases with increasing frequency. For higher frequencies, the anisotropy effects of the underlaying halfspace are barely detectable since the observed signal is composed mainly of the direct acoustic wave, resulting in the two domains being nearly indistinguishable.
\end{abstract}

Key words: Controlled source seismology; Guided waves; Seismic anisotropy.

\section{INTRODUCTION}

Normal modes in marine seismic acquisition and underwater acoustics refer to a family of asymptotic solutions of the wave equation when the source and receiver are placed in a water layer overlaying a solid half-space and the signal is recorded at a distance much larger than the water depth. The observed wavefield in this case consists of multiple water layer reflections and refractions and resulting horizontally propagating guided waves are dispersive. In exploration seismology, normal modes are often considered as a coherent noise and are removed at first stages of data processing (Boiero et al. 2013). In their excellent paper, Landrø \& Hatchell (2012) revisit the normal-modes theory and stress the potential use of the guided waves for shallow-sediments characterization. They demonstrate how to extract the normal modes from long-offset seismic data and perform a very simple velocity inversion. We direct the reader to the aforementioned reference for a historical review and the normal-modes theory in acoustic and elastic isotropic environments. Boiero et al. (2013) perform a combined surface- and guided-wave inversion for near-surface velocity model building demonstrating the usefulness of the part of the wavefield which is most of the time considered noise. The authors conclude that the discussed procedure can potentially improve a velocity model and results in a subsurface image of higher quality. The guided-wave inversion can also be incorporated into full waveform inversion (FWI) scheme to improve its accuracy in near-surface and overall efficiency as was demonstrated by Wiarda et al. (2014) and Hou et al. (2018). Another useful application of the normal modes is discussed in Landrø \& Amundsen (2011). The authors propose to use the guided wave signal in 4-D sense to detect shallow gas and $\mathrm{CO}_{2}$ leakage.

Landrø \& Hatchell (2012) mention that ocean-bottom sediments need not to be isotropic. Sidler \& Holliger (2010) use modeling to investigate the VTI anisotropy of shallow marine sediments and conclude that the $P$-wave anisotropy is benign but nevertheless non-zero; the $S$-wave anisotropy, on the other hand, is more prominent. Skopintseva et al. (2013) assume the sediments exhibit polar anisotropy (transversely isotropic with vertical symmetry axis, or VTI) and analyse the influence of the anisotropy parameters on normal-mode propagation. It has to be noted that normal modes in VTI case are first presented in Anderson (1961), however, due to the notation, derived expressions are not explicit. Ivanov \& Stovas (2017a) extend the findings of the aforementioned authors by incorporating azimuthal anisotropy described by an orthorhombic model. Unfortunately, derived orthorhombic period equation is not correct. In this work, we obtain a correct period equation in elastic orthorhombic case, providing detailed procedure and subsequently analyse the normal-mode propagation in orthorhombic media. 


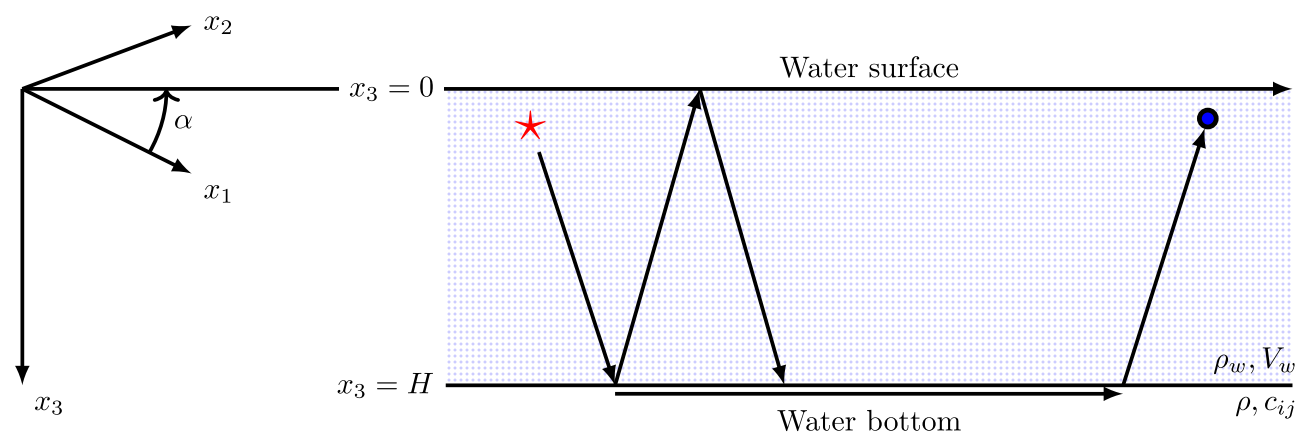

Figure 1. Sketch of the normal mode propagation. The star and the circle illustrate positions of a seismic source and a receiver, respectively. Parameters of the water layer, density $\rho_{w}$ and seismic velocity in water $V_{w}$, and of the elastic half-space, density $\rho$ and elastic stiffnesses $c_{i j}$, are displayed. $\alpha$ is the wave vector azimuth.

Although, one might argue that azimuthal anisotropy in water-bottom sediments is not feasible and it might be difficult to separate the effects associated with azimuthally varying water depth and heterogeneous sediments from actual anisotropy, a number of references indicate that the idea might not be as implausible as it seems. In gas-hydrate bearing sediments, one can encounter subvertical fracture systems (Lee \& Collett 2009; Cook et al. 2010; Sriram et al. 2013). Assuming transversely isotropic sediments with horizontal symmetry axis (HTI), Kumar et al. (2006) quantify the anisotropy to be around 15 percent (difference between the $P$-wave propagation along the symmetry axis and in orthogonal direction). Sriram et al. (2013) mention in their conclusions that they observe intersecting fracture systems and propose to use lower symmetry models such as orthorhombic. Tonegawa et al. (2015) observe the azimuthal anisotropy in the northwestern Pacific outside Japan and conclude that it is caused by the aligned fractures due to the tensional stress in the vicinity of the subduction zone.

The rest of the paper is organized as follows. First, we derive the exact period equation (provides the normal-mode phase velocity dependence upon frequency) in elastic orthorhombic case. Second, we obtain the frequency-dependent group velocity vector, which, in anisotropic media, is generally not parallel to the phase velocity vector. Third, we suggest two simplifications to the period equation which result in more practical expressions from the inversion standpoint. Finally, we provide numerical examples to demonstrate the anisotropy effects on the kinematics of normal-mode propagation.

\section{THEORY}

\subsection{The exact period equation}

Normal modes are a family of solutions of the wave equation able to describe horizontal propagation of seismic waves in a water layer overlaying an elastic half-space. Horizontal propagation is a consequence of multiple reflections and refractions in the water layer which acts as a wave guide (Landrø \& Hatchell 2012; Kazei et al. 2015). Although the normal modes propagate horizontally in the water layer, their signatures are influenced by the underlying half-space and are frequency dependent. In this study, the orthorhombic half-space is considered. Sketch of the normal-mode propagation along with the problem parametrization is shown in Fig. 1.

First, the wave propagation in orthorhombic half-space is considered. The component-wise wave equation for displacement vector $\mathbf{u}=\left(u_{1}, u_{2}, u_{3}\right)^{T}$ written for waves propagating in an elastic orthorhombic medium has the following form:

$c_{11} \partial_{1}^{2} u_{1}+c_{66} \partial_{2}^{2} u_{1}+c_{55} \partial_{3}^{2} u_{1}+\left(c_{12}+c_{66}\right) \partial_{12}^{2} u_{2}+\left(c_{13}+c_{55}\right) \partial_{13}^{2} u_{3}=\rho \partial_{t}^{2} u_{1}$,

$c_{66} \partial_{1}^{2} u_{2}+c_{22} \partial_{2}^{2} u_{2}+c_{44} \partial_{3}^{2} u_{2}+\left(c_{12}+c_{66}\right) \partial_{12}^{2} u_{1}+\left(c_{23}+c_{44}\right) \partial_{23}^{2} u_{3}=\rho \partial_{t}^{2} u_{2}$,

$c_{55} \partial_{1}^{2} u_{3}+c_{44} \partial_{2}^{2} u_{3}+c_{33} \partial_{3}^{2} u_{3}+\left(c_{13}+c_{55}\right) \partial_{13}^{2} u_{1}+\left(c_{23}+c_{44}\right) \partial_{23}^{2} u_{2}=\rho \partial_{t}^{2} u_{3}$,

where we express second-order partial derivatives in space as $\partial_{i j}^{2}=\partial^{2} / \partial x_{i} \partial x_{j}$ and in time as $\partial_{t}^{2}=\partial^{2} / \partial t^{2} ; c_{i j}$ are the orthorhombic stiffness coefficients. We seek a solution of the wave equation in the form of a plane wave propagating horizontally and expressed as

$\mathbf{u}=\mathbf{U}_{z}\left(x_{3}\right) e^{i\left(\omega t-k_{1} x_{1}-k_{2} x_{2}\right)}$,

where $\mathbf{k}=\left(k_{1}, k_{2}\right)^{T}$ is the wave vector, $\omega$ is a cyclic frequency, and, in order to account for vertically inhomogeneous medium, the depthdependent amplitude vector $\mathbf{U}_{z}$ is taken as

$\mathbf{U}_{z}\left(x_{3}\right) \equiv\left[U_{1}, U_{2},-i U_{3}\right]^{T} e^{-v x_{3}}$,

where coefficient $v$, that controls the amplitude attenuation with depth, is unknown at this step. Negative imaginary unit in front of $U_{3}$ in expression (3) is introduced to indicate the attenuation along the vertical. The magnitude of the wave vector, also known as the wave number, is related to the frequency as

$|\mathbf{k}|^{2}=k^{2}=k_{1}^{2}+k_{2}^{2}=\omega^{2} / c^{2}$, 
where $c$ is an unknown phase velocity of the plane wave. After substitution of the trial solution in the wave eq. (1) and subsequent simplification, we get in matrix form,

$\mathbf{G U}=\left[\mathbf{M}-\rho \omega^{2} \tilde{\mathbf{I}}\right] \mathbf{U}=0$,

where

$\mathbf{M}=\left[\begin{array}{ccc}c_{11} k_{1}^{2}+c_{66} k_{2}^{2}-c_{55} v^{2} & \left(c_{12}+c_{66}\right) k_{1} k_{2} & -\left(c_{13}+c_{55}\right) k_{1} v \\ \left(c_{12}+c_{66}\right) k_{1} k_{2} & c_{66} k_{1}^{2}+c_{22} k_{2}^{2}-c_{44} v^{2} & -\left(c_{23}+c_{44}\right) k_{2} v \\ -i\left(c_{13}+c_{55}\right) k_{1} v & -i\left(c_{23}+c_{44}\right) k_{2} v & -i\left(c_{55} k_{1}^{2}+c_{44} k_{2}^{2}-c_{33} v^{2}\right)\end{array}\right]$,

$\tilde{\mathbf{I}}=\left[\begin{array}{ccc}1 & 0 & 0 \\ 0 & 1 & 0 \\ 0 & 0 & -i\end{array}\right]$

and $\mathbf{U}=\left(U_{1}, U_{2}, U_{3}\right)^{T}$. Eq. (5) is satisfied if either $\operatorname{det} \mathbf{G}=0$ or $\mathbf{U}=0$. Since the latter is the trivial solution, the former equation is considered. It is natural to transform eq. (5) to cylindrical coordinates,

$k_{1}=k \cos \alpha, \quad k_{2}=k \sin \alpha, \quad k=\frac{\omega}{c}$,

where $\alpha$ is the phase azimuth (see Fig. 1). After the transformation, equation $\operatorname{det} \mathbf{G}=0$ is a bi-cubic polynomial equation with respect to $v / k$, and, defining

$\tilde{v}=\frac{v}{k}$,

it reads

$A_{6} \tilde{v}^{6}+A_{4} \tilde{v}^{4}+A_{2} \tilde{v}^{2}+A_{0}=0$,

where coefficients $A_{J}=A_{J}\left(c_{i j}, \alpha, \rho, c\right), J=0,2,4,6$ are given in Appendix A. Eq. $(10)$ is solved algebraically to obtain three roots, $\tilde{v}_{N}^{2}, N=$ $P, S_{1}, S_{2}$, that correspond to the three wave modes that exist in an elastic anisotropic solid-quasi-compressional and two quasi-shear. We do not present explicit expressions for $\tilde{v}_{N}$ due to their bulkiness, however, it is important to note that $\tilde{v}_{N}$ depend on the unknown phase velocity $c$.

The final solution of the wave equation in the orthorhombic half-space will then include three wave-modes propagating horizontally and attenuating with depth, each mode having different horizontal displacement and attenuation coefficient:

$\mathbf{u}^{\mathrm{ORT}}=\left[\mathbf{U}^{(P)} e^{-v_{P} x_{3}}+\mathbf{U}^{\left(S_{1}\right)} e^{-v_{S_{1}} x_{3}}+\mathbf{U}^{\left(S_{2}\right)} e^{-v_{S_{2}} x_{3}}\right] e^{i\left(\omega t-k_{1} x_{1}-k_{2} x_{2}\right)}$,

where $\mathbf{U}^{(N)}, N=P, S_{1}, S_{2}$ are unknown amplitude vectors of the three wave modes. To reduce the number of unknowns, we first transform the horizontal components of the amplitude vector into polar coordinates,

$U_{1}^{(N)}=U^{(N)} \cos \delta_{N}, \quad U_{2}^{(N)}=U^{(N)} \sin \delta_{N}$,

where $U^{(N)}$ is a radial component of the displacement amplitude, and $\delta_{N}$ is the polar angle that defines the azimuth of the horizontal projection of the polarization vector. Note, $\delta_{N}$ is generally different from $\alpha$ and $\alpha \pm \pi / 2$ in anisotropic media since displacement direction is not parallel or perpendicular to the phase propagation direction of quasi compressional and quasi shear waves, correspondingly. Relation between $\alpha$ and $\delta_{N}$ can, however, be established using any two equations of (5). From the first two, we obtain

$\tan \delta_{N}=\frac{q_{1}\left(c^{2} \rho+c_{55} \tilde{v}_{N}^{2}-c_{66} \sin ^{2} \alpha\right)+\frac{1}{2}\left(q_{2} q_{3}-c_{11} q_{1}\right) \sin 2 \alpha}{q_{2}\left(c^{2} \rho+c_{44} \tilde{v}_{N}^{2}-c_{66} \cos ^{2} \alpha\right)+\left(q_{1} q_{3}-c_{22} q_{2}\right) \sin ^{2} \alpha} \tan \alpha$,

where $q_{1}=c_{23}+c_{44}, q_{2}=c_{13}+c_{55}$ and $q_{3}=c_{12}+c_{66}$. Additionally, following the recipe of Anderson (1961), we express the radial component of the displacement amplitude of each wave mode in terms of the vertical component,

$U^{(N)}=\gamma_{N} U_{3}^{(N)}$

using third equation of (5). In eq. (14),

$\gamma_{N}=\frac{1}{\tilde{v}_{N}} \frac{c^{2} \rho+c_{33} \tilde{v}_{N}^{2}-c_{44} \sin ^{2} \alpha-c_{55} \cos ^{2} \alpha}{q_{2} \cos \alpha \cos \delta_{N}+q_{1} \sin \alpha \sin \delta_{N}}$,

and $U_{3}^{\left(P, S_{1}, S_{2}\right)}$ are the unknown amplitudes. Note that the expression for $\gamma_{N}$ simplifies to the one derived by Anderson (1961) when VTI media is considered ( $\alpha=\delta \equiv 0)$. Substituting the above expressions, eqs (12) and (14), in the total solution in the orthorhomibc half-space, eq. (11), the latter becomes

$\mathbf{u}^{\mathrm{ORT}}=\left[\begin{array}{c}U_{1} \\ U_{2} \\ U_{3}\end{array}\right] e^{i\left(\omega t-k_{1} x_{1}-k_{2} x_{2}\right)}=\sum_{N=P, S_{1}, S_{2}}\left[\begin{array}{c}\gamma_{N} U_{3}^{(N)} e^{-v_{N} x_{3}} \cos \delta_{N} \\ \gamma_{N} U_{3}^{(N)} e^{-v_{N} x_{3}} \sin \delta_{N} \\ -i U_{3}^{(N)} e^{-v_{N} x_{3}}\end{array}\right] e^{i\left(\omega t-k_{1} x_{1}-k_{2} x_{2}\right)}$. 
In the water layer, only compressional mode is present, however, since the layer is bounded, the solution is a superposition of up- and downgoing waves. Wave vector $\mathbf{k}$ is preserved in laterally homogeneous media to honor the Snell's law, meaning that the phase azimuth is preserved. Repeating similar derivations as above, we find for the water layer:

$v_{w}=k \tilde{v}_{w}=k \sqrt{1-\frac{c^{2}}{V_{w}^{2}}}$,

with $V_{w}$ being the velocity of the acoustic wave in water;

$\gamma_{w}=\tilde{v}_{w}^{-1}$

and the total solution of the wave equation in the water layer (taking into account that the displacement direction of the pure compressional wave is parallel to its propagation direction),

$\mathbf{u}^{\text {water }}=\left[\begin{array}{c}W_{1} \\ W_{2} \\ W_{3}\end{array}\right] e^{i\left(\omega t-k_{1} x_{1}-k_{2} x_{2}\right)}=\left[\begin{array}{c}\left(\grave{W} e^{-v_{w} x_{3}}+\dot{W}^{v_{w} x_{3}}\right) \cos \alpha \\ \left(\grave{W} e^{-v_{w} x_{3}}+\grave{W} e^{v_{w} x_{3}}\right) \sin \alpha \\ -i\left(\tilde{v}_{w} \grave{W} e^{-v_{w} x_{3}}-\tilde{v}_{w} \grave{W}^{v_{w} x_{3}}\right)\end{array}\right] e^{i\left(\omega t-k_{1} x_{1}-k_{2} x_{2}\right)}$,

where $\dot{W}$ and $\grave{W}$ are the unknown amplitudes of up- and downgoing solutions. Since $x_{3}$ is positive downwards, both solutions are characterized with an amplitude decay along the vertical. Different sign in front of vertical component of the amplitude of up- and downgoing solutions corresponds to different signs of $\tilde{v}_{w}$ : positive downwards and negative upwards.

In order to obtain unknown amplitudes $U_{3}^{(P)}, U_{3}^{\left(S_{1}\right)}, U_{3}^{\left(S_{2}\right)}, \grave{W}$, and $\dot{W}$, the boundary conditions are invoked,

$$
\begin{aligned}
\text { Free-surface condition : } & \left.\sigma_{33}\right|_{x_{3}=0}=0, \\
\text { Normal-stress continuity : } & \left.\sigma_{33}\right|_{x_{3}=H^{+}}=\left.\sigma_{33}\right|_{x_{3}=H^{-}}, \\
\text {Tangential stress vanishing in }\left[x_{1}, x_{3}\right] \text { plane : } & \left.\sigma_{13}\right|_{x_{3}=H^{+}}=0, \\
\text { Tangential stress vanishing in }\left[x_{2}, x_{3}\right] \text { plane : } & \left.\sigma_{23}\right|_{x_{3}=H^{+}}=0, \\
\text { Continuity of normal displacement : } & \left.U_{3}\right|_{x_{3}=H^{+}}=\left.W_{3}\right|_{x_{3}=H^{-}},
\end{aligned}
$$$$
\text { Tangential stress vanishing in }\left[x_{2}, x_{3}\right] \text { plane : }\left.\quad \sigma_{23}\right|_{x_{3}=H^{+}}=0 \text {, }
$$

where $H$ is the water-layer thickness, and $H^{-}$and $H^{+}$indicate media in which the function should be evaluated: minus sign corresponding to the water layer and plus sign - to the orthorhombic half-space. Free-surface condition (20a) results in up- and downgoing solutions in the water layer having opposing amplitudes since the free-surface reflection coefficient is assumed to be equal -1 ,

$\dot{W}=-\grave{W} \equiv W$.

Consequently, eqs (20b)-(20e) form a system of four equations with four unknowns. Written in matrix form, it reads

$\mathbf{B X}=0$,

where $\mathbf{X}=\left(U_{3}^{(P)}, U_{3}^{\left(S_{1}\right)}, U_{3}^{\left(S_{2}\right)}, W\right)^{T}$ is the vector of the unknowns and

$\mathbf{B}=\left[\begin{array}{cccc}e^{-k H \tilde{v}_{P}} & e^{-k H \tilde{v}_{S_{1}}} & e^{-k H \tilde{v}_{S_{2}}} & 2 \tilde{v}_{w} \cosh \left(k H \tilde{v}_{w}\right) \\ r_{P} e^{k H\left(\tilde{v}_{S_{1}}+\tilde{v}_{S_{2}}\right)} & r_{S_{1}} e^{k H\left(\tilde{v}_{P}+\tilde{v}_{S_{2}}\right)} & r_{S_{2}} e^{k H\left(\tilde{v}_{P}+\tilde{v}_{S_{1}}\right)} & 0 \\ S_{P} e^{k H\left(\tilde{v}_{S_{1}}+\tilde{v}_{S_{2}}\right)} & S_{S_{1}} e^{k H\left(\tilde{v}_{P}+\tilde{v}_{S_{2}}\right)} & S_{S_{2}} e^{k H\left(\tilde{v}_{P}+\tilde{v}_{S_{1}}\right)} & 0 \\ k l_{P} e^{-k H \tilde{v}_{P}} & k l_{S_{1}} e^{-k H \tilde{v}_{S_{1}}} & k l_{S_{2}} e^{-k H \tilde{v}_{S_{2}}} & 2 k \rho_{w} c^{2} \sinh \left(k H \tilde{v}_{w}\right)\end{array}\right]$,

where

$r_{N}=\cos \alpha+\gamma_{N} \tilde{v}_{N} \cos \delta_{N}$

$s_{N}=\sin \alpha+\gamma_{N} \tilde{v}_{N} \sin \delta_{N}$,

$l_{N}=c_{33} \tilde{v}_{N}-\gamma_{N}\left(c_{13} \cos \alpha \cos \delta_{N}+c_{23} \sin \alpha \sin \delta_{N}\right)$.

Eq. (22) is satisfied when $\operatorname{det} \mathbf{B}=0$, which, after simplification and using identity $\tanh (x)=-i \tan (i x)$, results in a period equation,

$$
\begin{aligned}
\tan \left(i k H \tilde{v}_{w}\right)= & \frac{i \tilde{v}_{w}}{c^{2} \rho_{w}}\left[\begin{array}{l}
\gamma_{P} \gamma_{S_{1}} \tilde{v}_{P} \tilde{v}_{S_{1}} \sin \left(\delta_{P}-\delta_{S_{1}}\right)+ \\
\gamma_{S_{2}} \tilde{v}_{S_{2}} \gamma_{S_{1}} \tilde{v}_{S_{1}} \sin \left(\delta_{S_{1}}-\delta_{S_{2}}\right)+ \\
\gamma_{S_{2}} \tilde{v}_{S_{2}} \gamma_{P} \tilde{v}_{P} \sin \left(\delta_{S_{2}}-\delta_{P}\right)
\end{array}\right]^{-1} \times \\
& {\left[\begin{array}{l}
\left(l_{S_{1}}-l_{S_{2}}\right) \gamma_{P} \tilde{v}_{P} \sin \left(\alpha-\delta_{P}\right)+l_{P} \gamma_{S_{1}} \gamma_{S_{2}} \tilde{v}_{S_{1}} \tilde{v}_{S_{2}} \sin \left(\delta_{S_{1}}-\delta_{S_{2}}\right)+ \\
\left(l_{S_{2}}-l_{P}\right) \gamma_{S_{1}} \tilde{v}_{S_{1}} \sin \left(\alpha-\delta_{S_{1}}\right)+l_{S_{1}} \gamma_{P} \gamma_{S_{2}} \tilde{v}_{P} \tilde{v}_{S_{2}} \sin \left(\delta_{S_{2}}-\delta_{P}\right)+ \\
\left(l_{P}-l_{S_{1}}\right) \gamma_{S_{2}} \tilde{v}_{S_{2}} \sin \left(\alpha-\delta_{S_{2}}\right)+l_{S_{2}} \gamma_{P} \gamma_{S_{1}} \tilde{v}_{P} \tilde{v}_{S_{1}} \sin \left(\delta_{P}-\delta_{S_{1}}\right)
\end{array}\right] }
\end{aligned}
$$


The obtained period equation allows one to study the guided-wave phase-velocity dispersion, that is $c=c(\omega)$. Right-hand side of eq. (25) depends, among model parameters, on the unknown phase velocity $c$ and not on the wavenumber, $k$. Hence, for a given phase velocity, one can compute the corresponding frequency using $k=\omega / c$ relation (eq. 8) in the left-hand side of the period equation. Since this computation requires taking an inverse tangent operation, there are multiple, in fact, infinite, solutions, corresponding to different normal modes. As discussed in details in Landrø \& Hatchell (2012) for isotropic elastic case, phase velocity $c$ take values between the sound velocity in water and the $P$-wave velocity in the underlaying half-space. In the infinite frequency limit, the phase velocity approaches the water velocity:

$\lim _{\omega \rightarrow \infty} c(\omega)=V_{w}$.

Physically, it means that the orthorhombic half-space has no influence on the normal-mode propagation and corresponding signal is just a direct wave in the water layer. In the opposite limit, when the guided-wave phase velocity is equal to the horizontal quasi-compressional wave velocity in the orthorhombic half-space (similar to the VTI case, as discussed in Skopintseva et al. 2013), the incidence angle of the acoustic wave is equal to the critical angle leading to $\tilde{v}_{P}=0$, and the corresponding frequency of the resulting guided wave is known as the cut-off frequency (minimum frequency for a given normal mode),

$\lim _{\omega \rightarrow \omega_{\text {cut-off }}} c(\omega)=V_{P}^{\text {hor }}$,

and each normal mode has a corresponding cut-off frequency. Value of the cut-off frequency increases with the mode number.

Since in orthorhombic media the horizontal velocity of the quasi-compressional wave is azimuthally dependent, the guided-wave phase velocity also varies with azimuth,

$V_{P}^{\text {hor }}(\alpha) \geq c(\omega, \alpha) \geq V_{w}$

where azimuthally dependent horizontal $P$-wave velocity $V_{P}^{\text {hor }}(\alpha)$ is obtained from the Christoffel equation for orthorhombic media. Please note, since both sides of the period eq. (25) are dimensionless, one is required to know the density contrast only and not individual densities of the media. Hence, the orthorhombic stiffness coefficients can be density normalized.

Eq. (25) simplifies to the period equation in elastic isotropic case (e.g. eq. 4.154 in Ewing et al. 1957),

$\tan \left(i k H \tilde{v}_{w}\right)=\frac{i \tilde{v}_{w} \rho c_{55}^{2}}{\tilde{v}_{P} \rho_{w} c^{4}}\left[4 \tilde{v}_{P} \tilde{v}_{S}-\left(2-\frac{c^{2}}{c_{55}}\right)^{2}\right]$,

when the corresponding parameters are substituted:

$c_{11}=c_{22}=c_{33}, \quad c_{44}=c_{55}=c_{66}, \quad c_{23}=c_{13}=c_{12}=c_{33}-2 c_{55}$,

resulting in

$\tilde{v}_{P}=\sqrt{1-\frac{c^{2}}{c_{33}}}, \quad \tilde{v}_{S_{1}}=\tilde{v}_{S_{2}}=\tilde{v}_{S}=\sqrt{1-\frac{c^{2}}{c_{55}}}$.

If the half-space is of VTI symmetry, eq. (25) simplifies to the one derived in Anderson (1961). Depending on which symmetry plane of the orthorhombic media is considered (both will result in the VTI period equation), the corresponding parameters should be used. It is important to note, the solution of the Christoffel eq. (10) will result in three roots in either of the symmetry planes, and the appropriate root selection is necessary to keep the quasi-shear wave solution that corresponds to the wave polarized within the symmetry plane (so-called SV wave).

\subsection{Group velocity}

Since propagation of the guided waves is dispersive in nature, one should expect phase and group (envelope) velocities to differ considerably. Moreover, in azimuthally anisotropic media, group velocity direction (defined as the energy propagation velocity) might differ from the phase velocity direction, as illustrated in Fig. 2. To the best of the authors knowledge, the problem of deriving the group velocity vector of the normal modes in azimuthally anisotropic media has not been solved yet. A very similar, although, significantly more complicated problem of the wave propagation in an azimuthally anisotropic free plate, also known as Lamb's problem, has been studied in details (Neau 2003; Wang \& Yuan 2007; Glushkov et al. 2014). Group velocity vector, denoted as g in derivations below, lays in the horizontal plane since the normal-mode propagation is horizontal. We express the group velocity using radial, $g_{r}$, and tangential, $g_{\alpha}$, components, the former being a projection of the group velocity onto the phase azimuth. Deviation between the phase and group velocity directions, often referred to as a steering angle (Neau 2003) or azimuthal lag (Koren \& Ravve 2017), is defined as

$\beta=\tan ^{-1} \frac{g_{\alpha}}{g_{r}}$.

Radial component of the group velocity is always positive, whereas the tangential component (and hence the steering angle) can take both positive and negative values. 


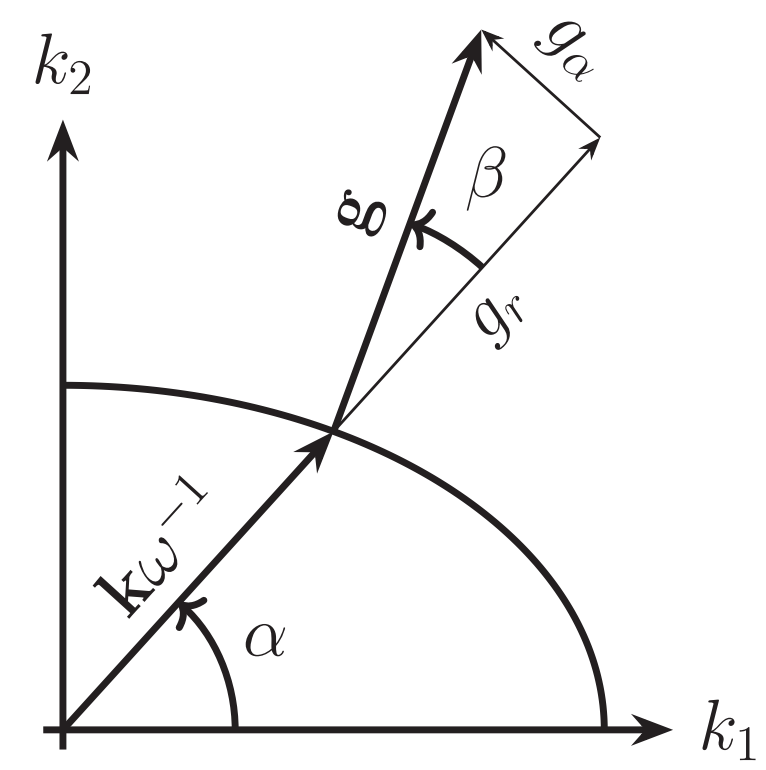

Figure 2. Schematic of the wave and group velocity vectors relation. $\mathbf{k} \omega^{-1}$ is the slowness vector defined by phase azimuth $\alpha, \mathbf{g}=\left\{g_{r}, g_{\alpha}\right\}$ is the group velocity vector (normal to the slowness surface), and the deviation between the two vectors is described by steering angle $\beta$.

The group velocity is calculated as (Auld 1973)

$\mathbf{g}=\frac{d \omega}{d \mathbf{k}}$,

where $\mathbf{k}=\{k \cos \alpha, k \sin \alpha\}$ is the wave vector. Let us denote the period equation, (25), compactly as $\Omega=\Omega(k, c=\omega / k, \alpha)=0$. Taking the full differential of the period equation at a fixed phase azimuth, one gets,

$d \Omega=\frac{\partial \Omega}{\partial \mathbf{k}} d \mathbf{k}+\left.\frac{\partial \Omega}{\partial \omega} d \omega\right|_{\alpha=\text { const }}$.

Although the tangential component $\left(k_{\alpha}\right)$ of the wave vector is zero, the dependence of $\Omega$ upon azimuth results in non-zero tangential component of the group velocity. Recalling the gradient definition in cylindrical coordinates,

$\frac{\partial \Omega}{\partial \mathbf{k}}=\left\{\frac{\partial \Omega}{\partial k}, \frac{\partial \Omega}{\partial k_{\alpha}}\right\}=\left\{\frac{\partial \Omega}{\partial k}, \frac{1}{k} \frac{\partial \Omega}{\partial \alpha}\right\}$,

and equating $d \Omega$ (eq. 34) to zero, the group velocity components are obtained,

$\mathbf{g}=-\frac{\frac{\partial \Omega}{\partial \mathbf{k}}}{\frac{\partial \Omega}{\partial \omega}}=\left\{g_{r}, g_{\alpha}\right\}=\left\{-\frac{\frac{\partial \Omega}{\partial k}}{\frac{\partial \Omega}{\partial \omega}},-\frac{\frac{1}{k} \frac{\partial \Omega}{\partial \alpha}}{\frac{\partial \Omega}{\partial \omega}}\right\}=\left\{-\frac{\frac{\partial \Omega}{\partial k}}{\frac{\partial \Omega}{\partial \omega}}, \frac{g_{r}}{k} \frac{\frac{\partial \Omega}{\partial \alpha}}{\frac{\partial \Omega}{\partial k}}\right\}$.

In the directions along the orthorhombic vertical symmetry planes, the steering angle is expected to be zero, since the phase velocity vector is parallel to the phase velocity surface normal along this directions. Outside the symmetry planes, however, the deviation can be significant. At the cut-off frequency, due to the critical incidence angle, the normal-mode signal is the result of constructive interference of head-wave multiples. The resulting phase velocity and the radial group velocity are equal to the horizontal velocity of the quasi-compressional wave in the orthorhombic medium (similar to the bulk waves), and the corresponding attenuation factor is zero, $\tilde{v}_{P}=0$, for all the azimuths. The transverse component of the group velocity and, hence, the steering angle reach maximum at the cut-off frequency, as we demonstrate in the numerical examples. Irrespective of the mode order, the phase and radial group velocity values in the corresponding frequency limits $(\omega$ $\rightarrow \omega_{\text {cut-off }}$ and $\left.\omega \rightarrow \infty\right)$ are preserved.

In addition to the derivation above, we would like to point out an important property of dispersive guided waves in anisotropic media. It is well-known that for bulk waves in elastic anisotropic media, the projection of the group velocity vector on the slowness direction is equal the phase velocity magnitude (Auld 1973; Grechka et al. 1999). Denoting the slowness vector with p, one gets

$\mathbf{p} \cdot \mathbf{g}=\frac{k}{\omega} g_{r}=\frac{g_{r}}{c}$.

For the bulk waves, the expression is equal to 1. For the normal modes, on the other hand, there is an additional term. Apart from eqs (36), one can use different formulation to calculate the radial group vector (e.g. eq. 1.27 in Neau 2003). For example, for a fixed phase azimuth, the radial group velocity component can be calculated, as shown in Landrø \& Hatchell (2012), using

$g_{r}=c+k \frac{d c}{d k}$. 
The second term can be obtained from the period equation, $\Omega=\Omega(k, c)$, after equating the full differential to zero,

$\frac{d c}{d k}=-\frac{\frac{\partial \Omega}{\partial k}}{\frac{\partial \Omega}{\partial c}}$.

Substituting eqs (38) and (39) in eq. (37), one arrives at

$\mathbf{p} \cdot \mathbf{g}=1-\frac{k}{c} \frac{\frac{\partial \Omega}{\partial k}}{\frac{\partial \Omega}{\partial c}}$.

The fact that the radial group velocity component is not equal the phase velocity is a distinctive feature of guided waves (moreover, unlike in the ray theory, the radial group velocity of the normal modes is smaller than the phase velocity).

\subsection{Acoustic and ellipsoidal approximations}

In certain cases, the quasi shear-wave velocity of sea-bottom sediments is expected to be considerably lower than the quasi compressional wave velocity (Landrø \& Hatchell 2012), hence, one can employ an acoustic orthorhombic approximation $($ Alkhalifah 2003$), V_{\mathrm{S} 0}=\sqrt{c_{55} / \rho}=0$. Acoustic orthorhombic media is characterized using six independent parameters, and, combining the notation used in Alkhalifah (2003) and Stovas (2015), we parametrize the half-space as follows:

$\begin{array}{ll}c_{11}=\rho V_{n 2}^{2}\left(1+2 \eta_{2}\right), & c_{12}=\rho V_{n 2} V_{n 1}\left(1+\eta_{x y}\right), \\ c_{22}=\rho V_{n 1}^{2}\left(1+2 \eta_{1}\right), & c_{13}=\rho V_{P 0} V_{n 2}, \\ c_{33}=\rho V_{P 0}^{2}, & c_{23}=\rho V_{P 0} V_{n 1},\end{array}$

where, $V_{P 0}$ is vertical $P$-wave velocity, $V_{n i}$ and $\eta_{i}$ are the normal moveout velocity and $P$-wave anellipticity coefficient defined in planes $\left[x_{2}\right.$, $\left.x_{3}\right], i=1$, and $\left[x_{1}, x_{3}\right], i=2$, respectively (Grechka $\&$ Tsvankin 1998, 1999). Parameter $\eta_{x y}$ is a cross-term anellipticity introduced in Stovas (2015), $\eta_{x y}=\sqrt{\left(1+2 \eta_{1}\right)\left(1+2 \eta_{2}\right) /\left(1+2 \eta_{3}\right)}-1$, where $\eta_{3}$ is the $P$-wave anellipticity defined in the horizontal plane.

The period equation in the acoustic case simplifies considerably since the Christoffel eq. (10) degrades to a quadratic polynomial equation and only one solution that corresponds to the quasi-compressional wave, $\tilde{v}_{P}$, exists. Boundary conditions on tangential-stress continuity, eqs (20c) and (20d), are satisfied in acoustic media. Repeating similar derivations as above using the remaining boundary conditions, one arrives at the acoustic period equation,

$\tan \left(i k H \tilde{v}_{w}\right)=\frac{-i \rho \tilde{v}_{w}}{\rho_{w} \tilde{v}_{P}}$

Substituting the expression for $\tilde{v}_{P}$, eq. (42) reads

$$
\begin{aligned}
& \tan \left(i k H \tilde{v}_{w}\right)=-\frac{i \rho V_{P 0} \tilde{v}_{w}}{\rho_{w} c} \times \\
& \sqrt{\frac{-1+\frac{V_{n 1}^{2}\left(1+2 \eta_{1}\right) \sin ^{2} \alpha}{c^{2}}+\frac{V_{n 2}^{2}\left(1+2 \eta_{2}\right) \cos ^{2} \alpha}{c^{2}}-\frac{V_{n 1}^{2} V_{n 2}^{2} E \sin ^{2} 2 \alpha}{4 c^{4}}}{c^{2}}},
\end{aligned}
$$

where $E=\left(\eta_{x y}+1\right)^{2}-\left(1+2 \eta_{1}\right)\left(1+2 \eta_{2}\right)$.

Since there exist no quasi-shear waves in the acoustic assumption, it is possible to obtain a concise expression for the cut-off frequency. As was mentioned before, propagation of the quasi-compressional wave at the cut-off frequency corresponds to the critical incidence and $\tilde{v}_{P}=0$, hence, from eq. (42),

$\omega_{\text {cut-off }}(\alpha)=\frac{\pi(2 n-1) V_{P}^{\text {hor }}(\alpha) V_{w}}{2 H \sqrt{\left[V_{P}^{\text {hor }}(\alpha)\right]^{2}-V_{w}^{2}}}$,

where $n=1,2, \ldots$ is the mode number. Similar equation in acoustic isotropic media can be found in Landrø \& Hatchell (2012, eq. 11).

Another useful simplification of the period equation can be derived by assuming $\eta_{i}=0$ in eq. (43). In this case, $P$-wave phase-velocity surface and the wavefront are ellipsoidal, and it requires only three parameters (three velocities along the ellipsoid's semi-axes) to describe the medium. The ellipsoidal period equation reads

$\tan \left(k H \sqrt{\frac{c^{2}}{V_{w}^{2}}-1}\right)=-\frac{\rho}{\rho_{w}} \sqrt{\frac{c^{2}}{V_{w}^{2}}-1}\left(\frac{V_{n 1}^{2} \sin ^{2} \alpha+V_{n 2}^{2} \cos ^{2} \alpha}{V_{P 0}^{2}}-\frac{c^{2}}{V_{P 0}^{2}}\right)^{-\frac{1}{2}}$. 
Simplicity of the above equation allows to explicitly obtain the group velocity expressions,

$$
\begin{aligned}
& g_{r}=c-c \frac{1-\frac{V_{w}^{2}}{c^{2}}}{1+\left[H k \frac{\rho_{w}}{\rho} \frac{V_{n \alpha}}{V_{P 0}}\left(1-\frac{c^{2}}{V_{n \alpha}^{2}}\right)^{3 / 2}\left(1-\frac{V_{w}^{2}}{V_{n \alpha}^{2}}\right)^{-1} \cos ^{-2} H k \sqrt{\frac{c^{2}}{V_{w}^{2}}-1}\right]^{-1}}, \\
& g_{\alpha}=\frac{\left(V_{n 1}^{2}-V_{n 2}^{2}\right) c^{-1} \sin \alpha \cos \alpha}{1+H k \frac{\rho_{w}}{\rho} \frac{V_{n \alpha}}{V_{P 0}}\left(1-\frac{c^{2}}{V_{n \alpha}^{2}}\right)^{3 / 2}\left(1-\frac{V_{w}^{2}}{V_{n \alpha}^{2}}\right)^{-1} \cos ^{-2} H k \sqrt{\frac{c^{2}}{V_{w}^{2}}-1} \times \frac{\frac{c_{w}^{2}}{V_{n \alpha}^{2}}-1}{V_{w}^{2}}-1},
\end{aligned}
$$

where $V_{n \alpha}^{2}=V_{n 1}^{2} \sin ^{2} \alpha+V_{n 2}^{2} \cos ^{2} \alpha$ is the phase-domain normal moveout velocity ellipse (Stovas 2015 ). To obtain the group velocity values at the cut-off and infinite frequencies, one has to take the corresponding limits of the expressions above, namely,

$$
\begin{aligned}
\left.\left\{g_{r}, g_{\alpha}\right\}\right|_{\omega \rightarrow \omega_{\text {cut-off }}} & =\lim _{c \rightarrow V_{n \alpha}}\left\{g_{r}, g_{\alpha}\right\}=\left\{V_{n \alpha}, \frac{V_{n 2}^{2}-V_{n 1}^{2}}{V_{n \alpha}} \sin \alpha \cos \alpha\right\}, \\
\left.\left\{g_{r}, g_{\alpha}\right\}\right|_{\omega \rightarrow \infty} & =\lim _{c \rightarrow V_{w}}\left\{g_{r}, g_{\alpha}\right\}=\left\{V_{w}, 0\right\} .
\end{aligned}
$$

One can note that at the cut-off frequency, the deviation direction of the group velocity vector from the phase velocity vector, expressed through the sign of the transverse component and, hence, of the steering angle, is controlled by the difference between the horizontal velocities along the orthorhombic symmetry planes. The expression suggests that in the ellipsoidal orthorhombic media, the group velocity vector is inclined (with respect to the phase velocity vector) towards the faster semi-axis of the horizontal velocity ellipse. Fig. 2 supports that observation and offers a clear geometrical explanation. In more realistic cases (acoustic and elastic orthorhombic models), the relation between the group and phase velocity vectors is more complicated and one cannot establish concise expressions as shown in eq. (47).

As easily observed from eq. (45), simplification to isotropic medium, achieved by setting $V_{n 2}=V_{n 1}=V_{P 0}$, leads to the well-known acoustic period equation (Ewing et al. 1957). The ellipsoidal approximation can be a starting point for the inversion if ocean-bottom sediments are expected to be azimuthally anisotropic and long-offset full-azimuth data exist for an area under investigation. Please note, both acoustic and ellipsoidal approximations of the period equation match the ones derived in Ivanov \& Stovas (2017a).

\section{NUMERICAL EXAMPLES}

Since influence of isotropic and transversely isotropic parts of the period equation as well as water layer properties have been extensively studied in existing literature (Landrø \& Hatchell 2012; Skopintseva et al. 2013), we direct our attention to azimuthal anisotropic effects. In particular, we numerically study the azimuthal dependence of the group velocity vector magnitude and direction for different frequencies, group velocity minimum value and its corresponding frequency, and the cut-off frequency. The stiffness tensor values for the examples are taken from Schoenberg \& Helbig (1997) and correspond to a vertically fractured transversely isotropic (VFTI) shale obtained using a linear-slip model. The density-normalized stiffness coefficients read $c_{11}=3.89, c_{22}=4.54, c_{33}=3.24, c_{44}=0.1, c_{55}=0.09, c_{66}=0.11$, $c_{23}=3.19, c_{13}=3.65$ and $c_{12}=3.24 \mathrm{~km}^{2} \mathrm{~s}^{-2}$. In the notation of Tsvankin (1997), the anisotropy parameters read $V_{P 0}=1.8 \mathrm{~km} \mathrm{~s}^{-1}, V_{S 0}=$ $0.3 \mathrm{~km} \mathrm{~s}^{-1}, \varepsilon^{(1)}=0.2, \delta^{(1)}=0.05, \varepsilon^{(2)}=0.1, \delta^{(2)}=0.2, \delta^{(3)}=-0.1, \gamma^{(1)}=0.15, \gamma^{(2)}=0.05$. Density ratio is $\rho / \rho_{w}=1.56$, water layer velocity and thickness are $V_{w}=1.485 \mathrm{~km} \mathrm{~s}^{-1}$ and $H=0.075 \mathrm{~km}$, respectively. Velocities along the orthorhombic horizontal symmetry axes are then $V_{11}=1.97$ and $V_{22}=2.13 \mathrm{~km} \mathrm{~s}^{-1}$.

Fig. 3 demonstrates the phase and group dispersion curves of the first four modes obtained by solving the period equation along different phase azimuths. The strong azimuthal variation of phase and group velocity values at the cut-off frequency is caused by the azimuthal velocity variation in the orthorhombic horizontal symmetry plane. One can also observe the azimuthal variation of the radial group velocity minimum. Besides, the minimum of the group velocity tends to take lower values for higher cutoff velocity values - the group velocity is 'stretched' vertically with increasing azimuth. The effect is stronger for higher-oder modes. Tangential component of the group velocity reaches its maximum at the cut-off frequency and rapidly decreases to zero with increasing frequency. The tangential component of the group velocity takes both positive and negative values, meaning that for some frequencies along certain azimuths, the group and the phase vectors can be perfectly aligned. It also results in different frequencies having different sign of the steering angle along one azimuth. Phase and radial-group velocity curves of the first normal mode along different azimuths are superimposed in a single graph in Fig. 4.

In Fig. 5, azimuthal variation of the phase and group velocities is shown for a number of fixed frequencies. Since in azimuthally anisotropic media phase and group velocity directions need not to be same (due to the curvature of a slowness surface), one has to consider phase and group azimuths separately (phase and solid lines in the figure, respectively). In orthorhombic media, due to the mirror symmetry with respect to the three orthogonal planes, phase and group velocities and corresponding directions are equal along the symmetry planes intersections (there are exceptions, but that is outside the scope of this paper), which, in the problem above, happens at the cut-off frequency along $0^{\circ}$ and $90^{\circ}$ azimuth. One can observe that the maximum difference between velocities (phase, group radial, and group transverse) in phase and group domains, happens, as expected, when the group transverse component is fairly large (mid-range in azimuth, low frequency). 

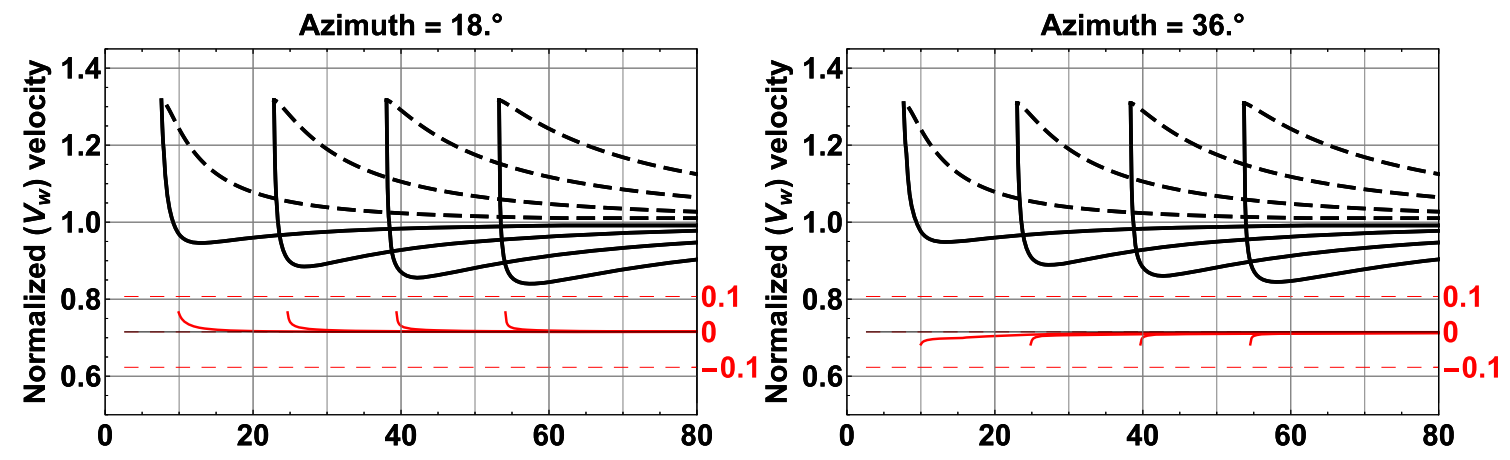

Azimuth $=36^{\circ}$

Azimuth $=5^{\circ} .^{\circ}$
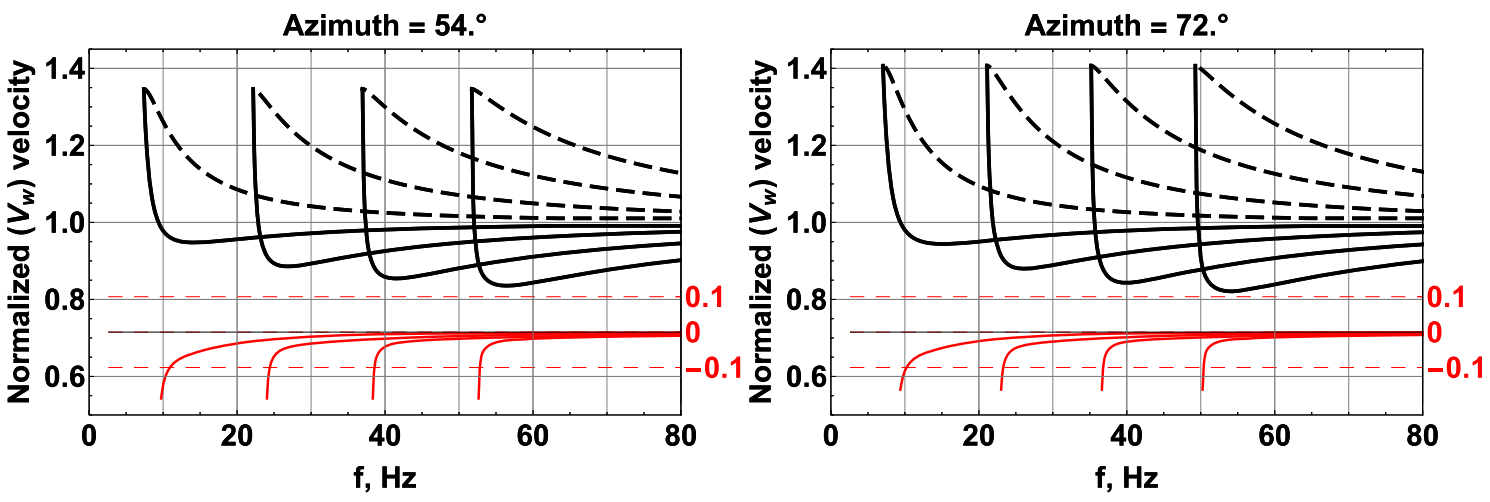

Figure 3. Dispersion curves of phase (dashed black), radial (solid black) and tangential (solid red) group velocities normalized to the water layer velocity along four different azimuths. First four modes are shown. Phase azimuth direction is specified. Note, values of the tangential group velocity are shown on the right axis, the scale between the left and the right axes are preserved.

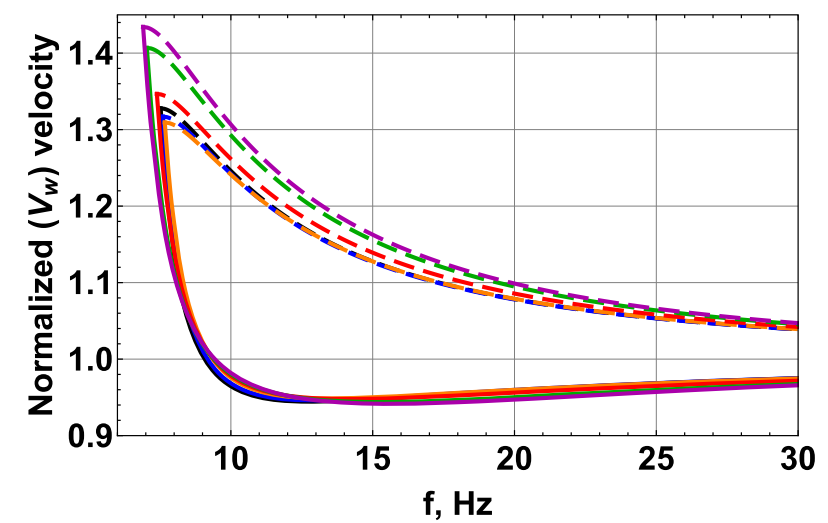

Figure 4. Dispersion curves of phase (dashed) and radial-group (solid) velocities normalized to the water layer velocity along six different azimuths: $0^{\circ}$ (black), $18^{\circ}$ (blue), $36^{\circ}$ (orange), $54^{\circ}$ (red), $72^{\circ}$ (green) and $90^{\circ}$ (magenta). Only first mode is shown.

The minimum difference, on the other hand, is when azimuthal direction is close to either of the symmetry directions $\left(0^{\circ}\right.$ and $\left.90^{\circ}\right)$. The difference between the domains is only significant (in fact, notable) for the frequencies close to the cut-off value, and the higher the frequency, the smaller the difference. To further stress this point, we demonstrate the steering angle dependence on the phase azimuth in Fig. 6 for different frequencies. Azimuthal variation of the cut-off frequency and the frequency that corresponds to the radial-group velocity minimum is shown in Fig. 7 for the first mode.

\section{DISCUSSION}

Presented period equation for orthorhombic media is a natural extension of the existing knowledge to more complicated subsurface models. Although, at first, orthorhombic anisotropy might not sound as a substantial increase in complexity when discussing quasi-compressional wave propagation (compared to the commonly accepted transversely isotropic models), it is characterized by features that do not exist in high symmetry anisotropic models. In this section, we would like to discuss some of the points that are omitted in the present study, but should be considered when dealing with orthorhombic models. 

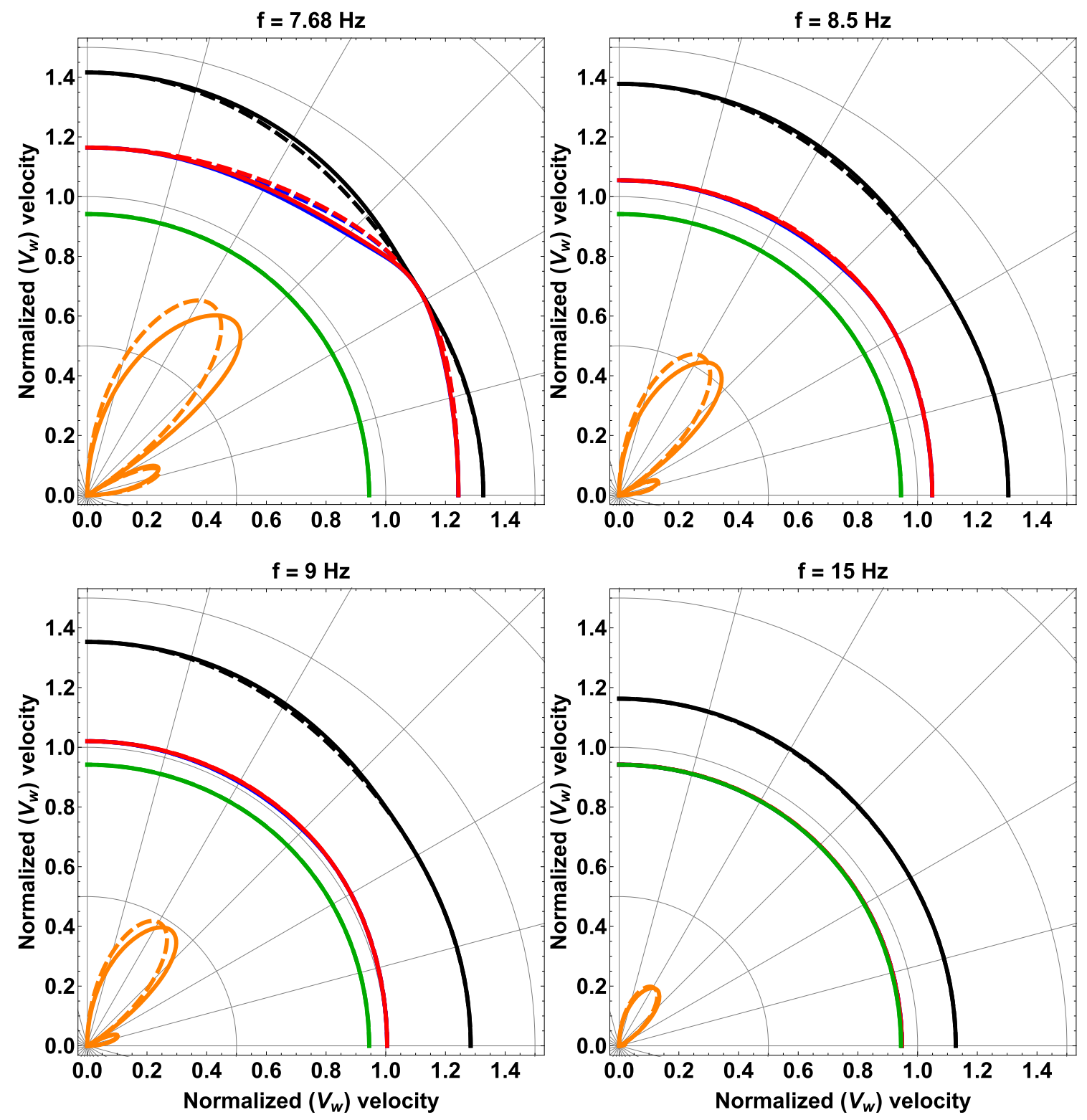

Figure 5. Azimuthal variation of phase (black), group radial (blue), group transverse (orange), group total (red) velocities and of the group velocity minimum (green) plotted against phase (dashed) and group (solid) azimuth directions for different frequencies. All velocities are normalized with the water velocity, the transverse component is exaggerated 5 times for demonstration purposes. A small loop on the transverse component along the azimuthal direction close to $15^{\circ}$ corresponds to positive velocity values, the larger loop is negative. Only first mode is shown.

First, orthorhombic models are characterized by existence (at least two and up to sixteen) of so-called shear-wave point singularitiesdirections along which the quasi-shear slowness sheets touch (Crampin 1981, 1991; Ivanov \& Stovas 2017b). These degeneracies lead to discontinuities in the corresponding wave surfaces, multiple shear wave arrivals (triplications), and caustics. The singularities are expected to affect the guided waves propagation, however, in the numerical model we consider, the maximum quasi-shear velocity is less than the water velocity and the resulting phase velocity range is not influenced by the shear-waves peculiarities.

Second, as has been pointed out for higher symmetry anisotropic and for elastic models, higher shear-wave velocities result in the wavenumber and, hence, the group velocity vector, being complex valued. That, in turn, means attenuation as discussed in Skopintseva et al. (2013). Taking into account the previous two points, one should expect a complicated combination of the shear-wave related effects based on the mutual relation between the water velocity, normal modes phase velocity, and quasi-shear slowness. Denoting $v_{S_{1}}$ and $v_{S_{2}}$ the phase velocities of the quasi-shear waves for a certain value of the guided wave phase velocity $c$ and phase azimuth $\alpha$, one should expect a number of different regimes, for example, $c>v_{S_{1}}$ and $c>v_{S_{2}}$ or $c>v_{S_{1}}$ and $c<v_{S_{2}}$ or $c<v_{S_{1}}$ and $c<v_{S_{2}}$, etc.

Third, the obtained period equation is derived in case of flat interfaces, infinite orthorhombic half-space, and infinite horizontal extension of the model. Although studying the guided-wave propagation in these simplified settings is instructive and influence of individual parameters is easily isolated, for practical (inversion) applications, a more realistic model of layered seabed should be considered. Examples in isotropic 


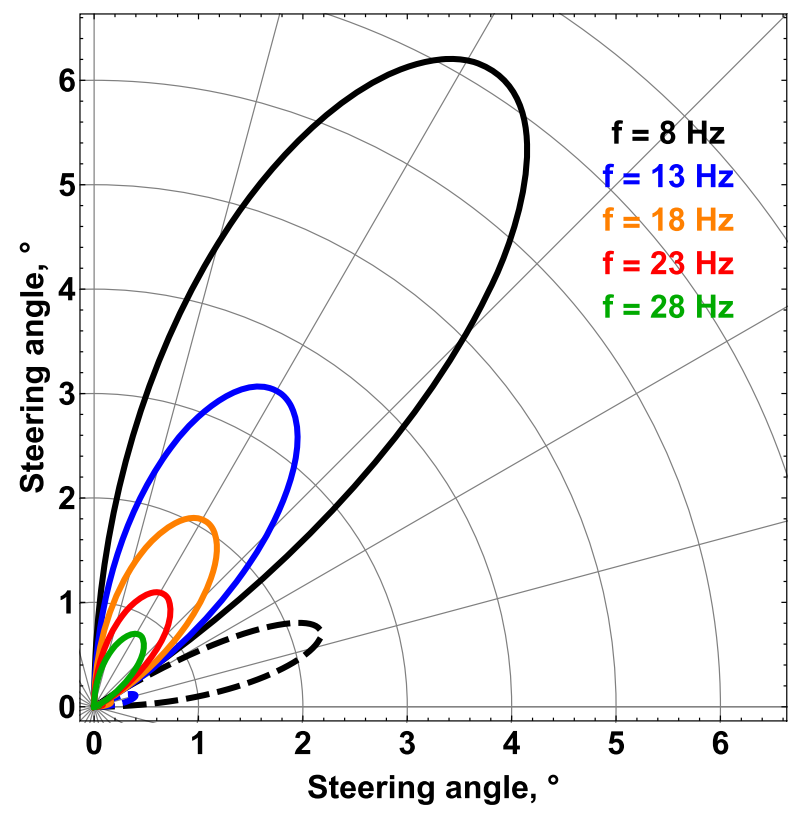

Figure 6. Azimuthal variation of the steering angle for different frequencies (the values are color-coded with the legend present in the plot). Solid and dashed lines correspond to negative and positive values, respectively. Only first mode is shown.

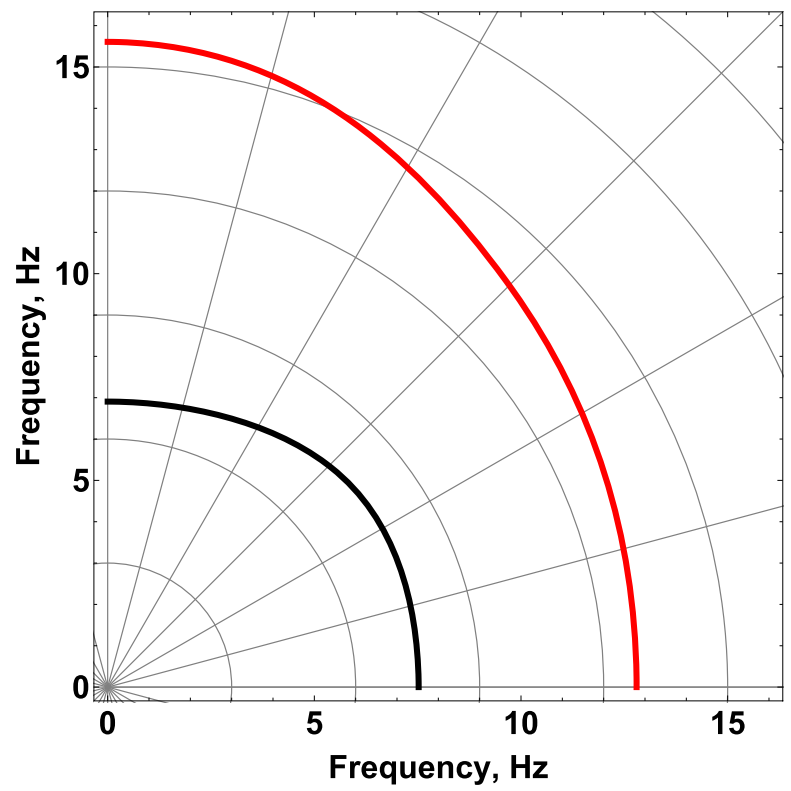

Figure 7. Azimuthal variation of the cut-off frequency (black) and the radial-group-velocity-minimum frequency (red). Only first mode is shown.

case are available (Landrø \& Amundsen 2011; Landrø \& Hatchell 2012; Boiero et al. 2013; Hou et al. 2018). Anisotropic cases are only available for a different application of non-destructive material testing (e.g. Wang \& Yuan 2007; Glushkov et al. 2014; Quintanilla et al. 2015).

Finally, as it was already mentioned, the period equation derived in Ivanov \& Stovas (2017a) for elastic orthorhombic media is erroneous. Although the functional form of that period equation is very different from eq. (25), the resulting dispersion curves are only slightly off when compared to the correct solution. To illustrate the discrepancy, we use the same numerical model as in the examples. Dispersion curves of phase and radial-group velocities are shown in Fig. S1, and tangential-group velocity curves are in Fig. S2. The provided acoustic and ellipsoidal approximations in Ivanov \& Stovas (2017a) are correct.

\section{CONCLUSIONS}

The period equation in fully elastic orthorhombic case is presented. Since number of independent parameters needed to fully describe such a system is beyond practical resolvability, acoustic and ellipsoidal orthorhombic approximations are developed. General approach towards 
the group velocity and azimuth calculation is outlined and applied to the ellipsoidal simplification leading to reasonably simple expressions. Using numerical test, the relation between phase and group domains in elastic orthorhombic case is studied. The overall conclusion is that the deviation between velocities and azimuths in these domains is the strongest for low frequencies and it rapidly decreases with increasing frequency. Hence, one should be able to safely assume that the phase and group azimuths are equivalent for frequencies sufficiently far from the cut-off frequency.

Although the evidence supporting orthorhombic symmetry of the ocean-bottom sediments is weak, the proposed simplifications of the exact period equation might be useful when accounting for the azimuthal dependence of the guided waves in a water layer. Correspondence between phase and group domains in low-symmetry anisotropic models is complicated and presented algebraic and numerical analysis is a starting point for development of more useful relationships.

\section{ACKNOWLEDGEMENTS}

Authors are grateful to the Editor Herve Chauris, and two reviewers, Zvi Koren and one anonymous. Their critical comments helped to improve the manuscript considerably. YI and AS acknowledge the Petromaks2 project for financial support, VK acknowledges KAUST for support.

\section{REFERENCES}

Alkhalifah, T., 2003. An acoustic wave equation for orthorhombic anisotropy, Geophysics, 68(4), 1169-1172.

Anderson, D.L., 1961. Elastic wave propagation in layered anisotropic media, J. geophys. Res., 66(9), 2953-2963.

Auld, B., 1973. Acoustic Fields and Waves in Solids, Vol. 1, WileyInterscience.

Boiero, D., Wiarda, E. \& Vermeer, P., 2013. Surface- and guided-wave inversion for near-surface modeling in land and shallow marine seismic data, Leading Edge, 32(6), 638-646.

Cook, A., Anderson, B., Malinverno, A., Mrozewski, S. \& Goldberg, D., 2010. Electrical anisotropy due to gas hydrate-filled fractures, Geophysics, 75(6), F173-F185.

Crampin, S., 1981. A review of wave motion in anisotropic and cracked elastic-media, Wave Motion, 3(4), 343-391.

Crampin, S., 1991. Effects of point singularities on shear-wave propagation in sedimentary basins, Geophys. J. Int., 107(3), 531-543.

Ewing, W.M., Jardetzky, W.S. \& Press, F., 1957. in Elastic Waves in Layered Media, McGraw-Hill.

Glushkov, E., Glushkova, N., Eremin, A. \& Lammering, R., 2014. Group velocity of cylindrical guided waves in anisotropic laminate composites, J. acoust. Soc. Am., 135(1), 148-154.

Grechka, V. \& Tsvankin, I., 1998. 3-D description of normal moveout in anisotropic inhomogeneous media, Geophysics, 63(3), 1079-92.

Grechka, V. \& Tsvankin, I., 1999. 3-D moveout inversion in azimuthally anisotropic media with lateral velocity variation; theory and a case study, Geophysics, 64(4), 1202-1218.

Grechka, V., Tsvankin, I. \& Cohen, J.K., 1999. Generalized Dix equation and analytic treatment of normal-moveout velocity for anisotropic media, Geophys. Prospect., 47(2), 117-148.

Hou, S., Haacke, R., Corbett, A. \& Wanczuk, M., 2018. Velocity model building with guided wave inversion, in Proceedings of the 80th EAGE Conference and Exhibition, p. We K 08, EAGE, Extended Abstracts.

Ivanov, Y. \& Stovas, A., 2017a. Normal modes in orthorhombic media, in Proceedings of the 79th EAGE Conference and Exhibition, p. We P6 03, EAGE, Extended Abstracts.

Ivanov, Y. \& Stovas, A., 2017b. S-wave singularities in tilted orthorhombic media, Geophysics, 82(4), WA11-WA21.

Kazei, V.V., Kashtan, B.M., Troyan, V.N. \& Mulder, W.A., 2015. FWI spectral sensitivity analysis in the presence of a free surface, in Proceedings of the 85th Annual International Meeting, pp. 1415-1419, SEG, Expanded Abstracts.

Koren, Z. \& Ravve, I., 2017. Fourth-order normal moveout velocity in elastic layered orthorhombic media - Part 2: Offset-azimuth domain, Geophysics, 82(3), C113-C132.
Kumar, D., Sen, M.K., Bangs, N.L., Wang, C. \& Pecher, I., 2006. Seismic anisotropy at Hydrate Ridge, Geophys. Res. Lett., 33(1), L01306.

Landrø, M. \& Amundsen, L., 2011. Using Water Layer Normal Modes to Detect Shallow Gas and CO2 Leakage, in EAGE Workshop on Permanent Reservoir Monitoring (PRM) - Using Seismic Data, pp. 10341, Extended Abstracts.

Landrø, M. \& Hatchell, P., 2012. Normal modes in seismic data-revisited, Geophysics, 77(4), W27-W40.

Lee, M.W. \& Collett, T.S., 2009. Gas hydrate saturations estimated from fractured reservoir at Site NGHP-01-10, Krishna-Godavari Basin, India, J. geophys. Res.: Solid Earth, 114(B7), B07102.

Neau, G., 2003. Lamb waves in anisotropic viscoelastic plates. Study of the wave fronts and attenuation, $P h D$ dissertation, Universite Bordeaux I, Bordeaux, France.

Quintanilla, F.H., Lowe, M.J.S. \& Craster, R.V., 2015. Modeling guided elastic waves in generally anisotropic media using a spectral collocation method, J. acoust. Soc. Am., 137(3), 1180-1194.

Schoenberg, M. \& Helbig, K., 1997. Orthorhombic media: modeling elastic wave behavior in a vertically fractured earth, Geophysics, 62(6), 19541974.

Sidler, R. \& Holliger, K., 2010. Seismic reflectivity of the sediment-covered seafloor: effects of velocity gradients and fine-scale layering, Geophys. $J$. Int., 181(1), 521-531.

Skopintseva, L.V., Landrø, M. \& Stovas, A., 2013. Normal modes in anisotropic VTI media, in Proceedings of the 75th EAGE Conference and Exhibition, Extended Abstracts, p. Th P03 07.

Sriram, G., Dewangan, P., Ramprasad, T. \& Rama Rao, P., 2013. Anisotropic amplitude variation of the bottom-simulating reflector beneath fracturefilled gas hydrate deposit, J. geophys. Res.: Solid Earth, 118(5), 22582274.

Stovas, A., 2015. Azimuthally dependent kinematic properties of orthorhombic media, Geophysics, 80(6), C107-C122.

Tonegawa, T., Fukao, Y., Fujie, G., Takemura, S., Takahashi, T. \& Kodaira, S., 2015. Geographical distribution of shear wave anisotropy within marine sediments in the northwestern Pacific, Prog. Earth planet. Sci., 2, 27.

Tsvankin, I., 1997. Anisotropic parameters and P-wave velocity for orthorhombic media, Geophysics, 62(4), 1292-1309.

Wang, L. \& Yuan, F.G., 2007. Group velocity and characteristic wave curves of Lamb waves in composites: modeling and experiments, Comp. Sci. Technol., 67(7), 1370-1384.

Wiarda, E.J., Shaw, S.A., Boiero, D., Gundersen, A. \& West, L.R., 2014. Integrating FWI with surface-wave inversion to enhance near-surface modelling in a shallow-water setting at Eldfisk, in Proceedings of the 76th EAGE Conference and Exhibition, p. Th E106 03, EAGE, Extended Abstracts. 


\section{SUPPORTING INFORMATION}

Supplementary data are available at $G J I$ online.

Figure S1. Dispersion curves of phase (black) and radial group (red) velocities normalized to the water layer velocity along six different azimuths. First four modes are shown. Solid lines are calculated using eq. (25) and dashed-using the period equation of Ivanov \& Stovas (2017a).

Figure S2. Dispersion curves of tangential group velocity normalized to the water layer velocity along four different azimuths. First four modes are shown. Solid lines are calculated using eq. (25) and dashed - using the period equation of Ivanov \& Stovas (2017a). Tangential group velocity is zero along $0^{\circ}$ and $90^{\circ}$ azimuths.

Please note: Oxford University Press is not responsible for the content or functionality of any supporting materials supplied by the authors. Any queries (other than missing material) should be directed to the corresponding author for the article.

\section{APPENDIX A: COEFFICIENTS OF THE BI-CUBIC POLYNOMIAL EQUATION}

Coefficients $A_{J}$ in eq. (10) have the following form:

$$
\begin{aligned}
A_{6}= & c_{33} c_{44} c_{55}, \\
A_{4}= & \cos ^{2} \alpha\left(c_{13}^{2} c_{44}-c_{33}\left(c_{11} c_{44}+c_{55} c_{66}\right)+2 c_{13} c_{44} c_{55}\right)+ \\
& \sin ^{2} \alpha\left(c_{23}^{2} c_{55}-c_{33}\left(c_{22} c_{55}+c_{44} c_{66}\right)+2 c_{23} c_{44} c_{55}\right)+ \\
& \rho c^{2}\left(c_{55} c_{33}+c_{44} c_{55}+c_{33} c_{44}\right), \\
A_{2}= & \left(c_{33}+c_{44}+c_{55}\right) \rho^{2} c^{4}+ \\
& {\left[c_{13}^{2}+2 c_{55} c_{13}-c_{11}\left(c_{33}+c_{44}\right)-c_{44} c_{55}-\left(c_{33}+c_{55}\right) c_{66}\right] \rho c^{2} \cos ^{2} \alpha+} \\
& {\left[c_{23}^{2}+2 c_{44} c_{23}-c_{22}\left(c_{33}+c_{55}\right)-c_{44} c_{55}-\left(c_{33}+c_{44}\right) c_{66}\right] \rho c^{2} \sin ^{2} \alpha+} \\
& {\left[c_{11}\left(c_{44} c_{55}+c_{33} c_{66}\right)-c_{13}\left(c_{13}+2 c_{55}\right) c_{66}\right] \cos ^{4} \alpha+} \\
& {\left[c_{22}\left(c_{44} c_{55}+c_{33} c_{66}\right)-c_{23}\left(c_{23}+2 c_{44}\right) c_{66}\right] \sin ^{4} \alpha+} \\
& {\left[2 c_{12} c_{13} c_{23}+2 c_{12} c_{55} c_{23}+2 c_{12} c_{13} c_{44}+2 c_{13} c_{66} c_{23}+\right.} \\
& c_{23}\left(c_{55} c_{66}-c_{11} c_{44}\right)+2 c_{13}\left(c_{44} c_{66}-c_{22} c_{55}\right)+2 c_{12}\left(c_{44} c_{55}-c_{33} c_{66}\right)+ \\
& \left.c_{11} c_{22} c_{33}+4 c_{44} c_{55} c_{66}-c_{33} c_{12}^{2}-c_{11} c_{23}^{2}-c_{22} c_{13}^{2}\right] \sin ^{2} \alpha \cos ^{2} \alpha, \\
A_{0}= & \left(c_{44} \sin ^{2} \alpha+c_{55} \cos ^{2} \alpha-\rho c^{2}\right) \times \\
& \left\{-\rho^{2} c^{4}+\left[c_{11}+c_{66}\right] \rho c^{2} \cos ^{2} \alpha+\left[c_{22}+c_{66}\right] \rho c^{2} \sin ^{2} \alpha-\right. \\
& \left.c_{11} c_{66} \cos ^{4} \alpha-c_{22} c_{66} \sin ^{4} \alpha-\left[c_{11} c_{22}-c_{12}\left(c_{12}+2 c_{66}\right)\right] \sin ^{2} \alpha \cos ^{2} \alpha\right\} .
\end{aligned}
$$

